Michael Schwarze (Konstanz)

\title{
„Un champ de bataille on ne peut plus réel“. Zur Referentialität von Distanz und Nähe in 14 von Jean Echenoz
}

\begin{abstract}
This paper addresses the production of referentiality in historical fiction. It analyses Jean Echenoz' World War I novel 14 (2012), by identifying textual patterns that generate effects of distance and proximity. These effects concern the metatextual dialogue the novel holds with conventional ways of representing this war, and they also relate to the actual historical past evoked by the novel. In this respect 14 performs a notable inversion. Historical fiction usually produces effects of distance in order to convey historical explanation and achieves historical concreteness by effects of proximity, whereas Echenoz endeavours to provide certain aspects of the war with explicative coherence, registering the material world with great concreteness. In many passages, on the other hand, his minimalist writing produces an ironic distance, which makes experiences of contingency aesthetically evident.
\end{abstract}

Seit 2014 wird in vielen europäischen Ländern mit politischen Feierstunden, Ausstellungen, wissenschaftlichen Kolloquien, öffentlichen Veranstaltungen und publizistischen Interventionen des Ersten Weltkriegs gedacht. In Frankreich und Deutschland steht dafür bis heute als Symbol der Name Verdun, die publizistische und mediale Erinnerung an den Beginn der zehn Monate dauernden Material- und Abnutzungsschlacht vor 100 Jahren hat dies kürzlich erneut eindrücklich vor Augen geführt.

Zum zentralen Stellenwert, den die Grande Guerre zumal in der öffentlichen Erinnerungskultur Frankreichs nach wie vor hat, trug wesentlich bei, dass der Krieg mit dem Waffenstillstand vom November 1918 keineswegs zu Ende war, sondern dass es in den zwanziger Jahren als dringende gesamtgesellschaftliche Aufgabe angesehen wurde, dem vorderhand Sinnlosen, dem grauenvollen Dahinschlachten während des Krieges nachträglich einen Sinn zuzuschreiben. Im Bereich der kulturellen Produktion kam dabei neben den bildenden Künsten und dem noch jungen Kino vor allem der Literatur die tragende Rolle zu. ${ }^{1}$ In Frankreich, auf das ich mich im Folgenden beschränken werde, waren es insbesondere hunderte von Romanen, die den Krieg meist im Sinn einer „nationalen Synthese“

1 Vgl. dazu Lindner-Wirsching 2004 und 2011.

DOI 10.1515/roja-2017-0009 
deuteten. Sie trugen so, wie jüngst konzise Carsten Kretschmann gezeigt hat, dazu bei, ihn für alle Überlebenden aufzuarbeiten und politisch operabel zu machen. ${ }^{2}$

Der Kriegsroman der 20er und 30er Jahre knüpfte dabei entfernt an eine beinahe 100 Jahre alte Tradition an: Diese bestand bekanntlich darin, dass die Gattung Roman in den ersten Jahrzehnten des 19. Jahrhunderts komplementär zum zweiten führenden Medium einer Reflexion geworden war, in dem sich ein grundlegender Wandel des Geschichtsverständnisses vollzog. ${ }^{3}$ Namentlich erinnert sei hier lediglich an die literarische Mode des historischen Romans, die ausgehend vom Vorbild Walter Scotts in den 1820er Jahren schnell auf das kontinentaleuropäische Publikum übergriff.

Blickt man an das andere Ende dieser literarhistorischen Zeitachse, in die Gegenwart, ist gerade in den als posthistorisch etikettierten letzten 35 Jahren eine auffällige „renarrativisation historique“ $\mathrm{zu}$ beobachten. ${ }^{4}$ Im Zuge dieser literarischen Rehistorisierung erweist sich der Roman unzweifelhaft als die Gattung, in der Geschichten von der Geschichte nach wie vor bevorzugt erzählt werden. Eindrücklich belegt dies - um beim Ersten Weltkrieg zu bleiben - die Zahl von circa 100 erschienenen Romanen, die Griet Theeten in einer grundlegenden Studie für den Zeitraum zwischen 1980 und 2000 gezählt hat. ${ }^{5}$ Zeitgenössische fiktionale Retextualisierungen der Grande Guerre werfen unweigerlich die Leitfrage auf, mit welchen Zielen und welchen Ergebnissen Romane im Abstand von vielen Jahrzehnten und mehreren Generationen einen Krieg wiedererzählen, über den die Kriegsromane der Zeugengeneration und die internationale Geschichtswissenschaft alles gesagt zu haben scheinen. Die Motivationen für die zeitgenössische Produktion sind weit gestreut und überlagern sich: Sie reichen von der kommerziellen Verwertung eines wohlfeilen Sensationalismus über die sentimentale Befriedigung eines erinnerungskulturellen Konsensus, mit dem unsere postheroische Kultur dem Phänomen Krieg vielfach begegnet, ${ }^{6}$ über investigative Romane, die unser Nicht-Wissen über die historische Vergangenheit publikumswirksam zu

2 Kretschmann 2014. Siehe zum Ersten Weltkrieg die hervorragend informierte Encyclopédie de la Grande Guerre: Audoin-Rouzeau/Becker ${ }^{2} 2012$.

3 Siehe dazu konzise Föcking 2002.

4 Siehe dazu Dominique Viarts (2009) vorzügliche mise au point „Nouveaux modèles de représentation de l'histoire en littérature contemporaine“ sowie die beiden „Introductions“ in: Rubino/Viart 2014, 11-40.

5 Vgl. Theeten 2009, 3. Verlässliche Zahlen für die letzten 15 Jahre liegen mir leider nicht vor.

6 So bescheinigt Pierre Schoentjes (2012, 980) der jüngsten Flut von französischen Romanen über den Ersten Weltkrieg einen „néo-réalisme sensationnaliste“. Sylvie Mikowski (2015, 42) beklagt gar eine generelle „pétrification de la mémoire collective de la Guerre dans des images codifiées“, gegen die Autoren wie Sebastian Barry und Jean Echenoz anschrieben. 
verringern suchen, ${ }^{7}$ bis hin zur Archäologie von historischen Familienkonstellationen, wie sie das Subgenre des récit de filiation seit den 1990er Jahren praktiziert. ${ }^{8}$ Der gemeinsame Nenner einer Vielzahl solcher Romane besteht darin, dass ihre Verfasser sich die Geschichte des Ersten Weltkrieges dezidiert von einem heutigen, bewusst subjektiven Standpunkt aus neu aneignen und sie in ethischer Absicht reinterpretieren. ${ }^{9}$ Sofern sie dabei ein historisches Erkenntnisinteresse im engeren Sinne verfolgen, liegt dieses meist darin, Wirklichkeitsanteile des Krieges zu profilieren, die in anderen Wirklichkeitsdiskursen unterbelichtet geblieben sind. Auf diese Weise versuchen sie, gleichsam komplementär die Ausdifferenzierung des historischen Diskurses über den Krieg literarisch voranzutreiben. ${ }^{10}$

1 Im Horizont dieses Segments der Gegenwartsliteratur, für die sich in der französischen Forschung der Begriff der littérature de l'extrême contemporain eingebürgert hat, ${ }^{11}$ liegt das Interesse dieses Aufsatzes. Anhand der Einzelfalluntersuchung eines Gegenwartsromans von Jean Echenoz geht er exemplarisch der weitreichenden Frage nach, wie der zeitgenössische Geschichtsroman mit den narrativen und fiktionsbildenden Mitteln, die er typischerweise intensiver als andere Formen der Geschichtsrede einsetzt, auf die historische Vergangenheit referiert. Dies setzt die theoretische Annahme voraus, dass die fiktionale Darstellung von Wirklichkeit nicht an die inzwischen hinfällige Trennung zwischen fiktiven und faktualen Gegenständen gebunden ist, sondern dass in ihr der Bezug auf res factae wie res fictae grundsätzlich gleichermaßen im Dienst einer plausiblen Rekonstruktion der historischen Wirklichkeit stehen kann. ${ }^{12}$

7 Als Beispiele hierfür können gelten Didier Daeninckx (1985): Le der des ders oder Pierre Lemaître (2013): Au revoir là-haut. Dass es sich dabei um Romane handelt, die partiell einen Kriminalplot mit Rätselstruktur haben, ist kein Zufall. Siehe dazu Gauyat 2009.

8 Prominente Familienarcheologien des Ersten Weltkriegs sind Claude Simon: L’Acacia (1989); Jean Rouaud: Les Champs d'honneur (1990); Pierre Bergounioux: L'Orphelin (1992).

9 In Anlehnung an Hartogs drittes Geschichtsregime des „présentisme“ schreibt Viart $(2009,18)$ dazu treffend: „Désormais le présent, continue l'historien, est omniprésent, double inféode à une dette envers le passé qu'il ne parvient à résorber, mais aussi envers le futur dont il se sent responsable au point d'endiguer ses propres actions.“ Vgl. dazu grundlegend Hartog 2003.

10 Siehe zu dieser in der Forschungsdiskussion vielfach konstatierten Intention und mit Bezug auf das intrikate Verhältnis zwischen historischem Roman und Historismus: Brecht 2002, insbesondere 425-436; in kulturwissenschaftlicher Perspektive: Assmann 2016. Die historische Entwicklung dieses Gedankens vom historischen Roman des 19. Jahrhunderts bis zum New Historicism entwickelt Mario Domenichelli 2006.

11 Der Begriff geht auf eine Sondernummer der Zeitschrift Po\&sie zurück, die 1987 von dem Schriftsteller und Kritiker Michel Chaillou herausgegeben wurde. Siehe dazu eingehend Viart 2000.

12 Vgl. dazu Schaeffer ${ }^{2} 2014$. 
In der Geschichtstheorie und der Theorie der Historiographie war die Referentialität der Geschichtsrepräsentation, ausgelöst durch die Thesen Hayden Whites, lange Gegenstand intensiver Forschungen gewesen. Whites provozierende, 1973 in der Studie Metahistory vertretene Auffassung, die Geschichtsschreibung sei wie die Literatur letzten Endes nichts anderes als eine „verbal fiction“, da ihre Erzählweise stets auf literarisch verbürgten Verfahren des emplotments beruhe, ${ }^{13}$ ließ die Referenz aller historischen Erzählung auf eine außersprachliche Wirklichkeit als ein problematisches Konstrukt erscheinen. ${ }^{14}$ Diesem ,narrativistischen“ Ansatz hat insbesondere der französische Geschichtstheoretiker Paul Ricœur prominent geantwortet, indem er für die Historiographie auf der Existenz referentieller Spuren beharrt, welche auf einem faktualen Wirklichkeitsbegriff fußen und ihr methodisches Fundament in der Quellenkritik haben. ${ }^{15}$

Die literaturwissenschaftliche Theoriebildung war ihrerseits im Kontext des linguistic turn und des New Historicism mit einer radikalen Infragestellung jeglicher Referenzfähigkeit des fiktionalen Erzählens konfrontiert. Wenn die Fiktion das, was sie referentiell $\mathrm{zu}$ repräsentieren vorgibt, letztlich erst selbst hervorbringt, hat dies zur Folge, dass sie keinerlei Anspruch auf Wahrheit erheben kann. Damit aber muss die Möglichkeit des Geschichtsromans, ein adäquates Mittel zur Rekonstruktion von Vergangenem zu sein, ad acta gelegt werden so die entsprechende Argumentation. Auf diese insbesondere in der nordamerikanischen Forschung vertretene These haben Ansgar Nünning, Daniel Fulda, Andreas Kablitz und andere geantwortet, indem sie eine kategoriale Differenz

13 White 1973, Zitat IX.

14 In dem grundlegenden 1978 erschienenen Aufsatz „The Historical Text as Literary Artefact“ heißt es dazu: „In point of fact, history [...] is made sense of in the same way that the poet or the novelist tries to make sense of it, i.e. by endowing what originally appears to be problematical and mysterious with the aspect of a recognizable, because it is a familiar, form. It does not matter whether the world is conceived to the real or only imagined; the manner of making sense of it is the same. “(White 1978, 81-100, hier 98) Siehe für eine kritische Bestandsaufnahme einer Narratologie der Geschichtsschreibung Fulda ${ }^{2} 2014$.

15 In seiner 2000 erschienenen mise au point La mémoire, l'histoire, l'oubli begegnet Ricœur dem von White aufgeworfenen Problem umfassend, indem er den Wirklichkeitsbezug der Geschichtsnarration durch das Zusammenspiel dreier operationaler Phasen des „régime historiographique“ gewährleistet sieht: Erst wenn die abschließende „literarische Formgebung“ („phase scriptuaire“) die Ergebnisse der vorangehenden Arbeitsstadien des „dokumentarischen Beweises“ und der „kausalen/finalen Erklärung“ der Dokumente berücksichtige, könne die Geschichtsdarstellung Anspruch auf historische Erkenntnis erheben. Pointiert fasst Ricœur die „spécificité de la référentialité en régime historiographique“ dabei unter dem Begriff „représentance“ wie folgt zusammen: „[...] c'est ensemble que scripturalité, explication compréhensive et preuve documentaire sont susceptibles d'accréditer la prétention à la vérité du discours historique“ (Ricœur 2000, 363). Siehe zur Debatte White-Ricœur-White Tengelyi 2010. 
zwischen der Vergangenheit und ihrer Textualisierung in Form von Geschichte geltend gemacht haben. ${ }^{16}$ Die Unterscheidung zielt darauf ab, dass die Vergangenheit an sich ein unstrukturiertes Geschehen darstellt, dem erst im Prozess der Vertextlichung (samt der mit ihr notwendigerweise verbundenen Verfahren der Selektion und Reduktion) die rekonstruktive Kohärenzstruktur zugeschrieben wird, die wir von der Geschichte erwarten. Das Vergangene muss demnach gleichsam erschrieben werden, um als Geschichte zu gelten. ${ }^{17}$

Meine Überlegungen teilen diesen Ansatz und betrachten daher die textuelle Verfasstheit der Geschichtserzählung als deren zentrales Konstituens. Für die Frage nach der Referentialität von Geschichtsromanen bedeutet dies methodisch, dass für ihre Beantwortung die Untersuchung der textuellen Verfahren, welche die literarische Fiktion zur Herstellung von Referenzeffekten einsetzt, von zentraler Bedeutung ist. Denn erst wenn fiktionsbildende und narrative Textmuster eines Romans im Hinblick auf ihre referenzstiftende Funktion analysiert werden, ist es möglich, die Frage zu beantworten, wie er historische Wirklichkeitserfahrungen modelliert. Diese theoretischen Einsichten jedoch haben zumal auf dem Feld der französischen littérature de l'extrême contemporain kaum zu einer kritischen Masse von literaturwissenschaftlichen Einzelfallanalysen geführt, die literarische Geschichtserzählungen gleichsam historiographisch lesen ${ }^{18}$ und die so die Identifikation von Textualisierungsmustern ermöglichen würden, mit denen literarische Texte gegebenenfalls in spezifischer Weise auf die historische Vergangenheit referieren. ${ }^{19}$ An diesem Desiderat setzt die folgende Textanalyse an.

Sie hat mit 14 von Jean Echenoz einen zeitgenössischen Roman von 2012 zum Gegenstand, der in 15 Miniatur-Kapiteln auf knapp 120 Seiten eine fiktive Geschichte der Grande Guerre erzählt. Sein Verfasser, Jahrgang 1947, gehört bekanntlich zu einem Segment von Romanciers, die in der Tradition eines prononciert experimentellen, selbstreflexiven Erzählens stehen, wie es in den 1950er und 60er Jahren die Vertreter des Nouveau Roman initiiert hatten. ${ }^{20} \mathrm{Zu}$ den Exponenten dieser seit den 1980er Jahren von Jérôme Lindon geförderten Autoren,

16 Siehe dazu Nünning 1999; Fulda 2002; Kablitz 2006. Methodisch liegen dem Folgenden insbesondere Fuldas Prämissen für die Systematisierung einer „strukturanalytischen Hermeneutik“ zugrunde, 44-49.

17 Ich übernehme diese Formulierung von Fulda 2002, 40, 45 passim.

18 Siehe dazu die Fallstudien in Rüth/Schwarze 2016.

19 Fulda konstatierte dieses Defizit bereits 2002 und führte als Begründung für die Scheu der Literaturwissenschaften die nach wie vor plausible These an, die Literaturwissenschaft verwende allzu häufig einen „ontologischen Fiktionsbegriff, der literarische Texte auf eine Weise von ihren außerliterarischen Diskursnachbarn trennt, die die konstitutive Rolle von Fiktionen bei jeder Geschichtskonstruktion mißachtet.“ (46f.)

20 Vgl. hierzu Dugast 2009. 
die auch die nouveaux auteurs de Minuit genannt werden und die sich einer intensiven Rezeption erfreuen, ${ }^{21}$ zählen neben Echenoz Jean-Philippe Toussaint, Eric Chevillard et Christian Gailly sowie seit den 1990ern Marie N'Diaye, Christian Oster und Laurent Mauvignier. Die institutionelle Klammer dieser Autoren stellt das Pariser Verlagshaus Les Editions de Minuit und dessen konsequentes Verlagsprogramm dar, dem auch bereits die nouveaux romanciers Alain RobbeGrillet, Michel Butor und Nathalie Sarraute eng verpflichtet waren. Der dezidierte Anspruch der heute prägenden Minuit-Autoren, in ihren écritures das experimentelle Erbe der vorangehenden Minuit-Generation fortzuschreiben, macht ihre Texte, sofern es sich um Geschichtsfiktionen handelt, zu einem spezifisch französischen Beobachtungsfeld für die hier interessierende Fragestellung. ${ }^{22}$

2 Liest man $14,{ }^{23}$ stellt sich zunächst freilich leicht der Eindruck einer durchaus konventionellen Kriegserzählung ein. Dies liegt an seiner für einen Kriegsroman typischen Plotstruktur: ${ }^{24}$ Im Mittelpunkt der Handlung, die im August 1914 in der Region von Nantes im Moment der Mobilmachung einsetzt, stehen die Sprösslinge zweier assoziierter Industriellenfamilien, die als Schuhfabrikanten zu Geld und Ansehen gelangt sind. Die ungleichen Brüder Anthime und Charles werden im August 1914 zur Infanterie einberufen und an die Front im Osten Frankreichs verlegt. Mit ihnen ziehen drei „camarades de pêche et de café“ (15) von Anthime in den Krieg: der Schlachterjunge Padioleau, Bossis, seines Zeichens Abbrucharbeiter, sowie der Sattler Arcenel. Charles und Anthime lassen mit Blanche die einzige Tochter der Inhaberfamilie des Unternehmens zurück; beide lieben sie und von einem der beiden ist sie schwanger. In dieser amourösen Dreiecksgeschichte, die ausgesprochen typisch für Kriegsromane ist, ist der selbstbewusste Charles der Versprochene, während Anthime Blanche im Geheimen liebt. Der Roman erzählt die Erlebnisse und Schicksale dieser sechs Figuren an und hinter der Front in einer Abfolge lose miteinander verknüpfter Kapitel. Echenoz verfährt dabei geradezu klassisch, indem er dichte Episoden konstruiert, die vorrangig das Ziel haben, das Personal in einer möglichst großen Zahl von für den Krieg repräsentativen Schlüsselszenen zu zeigen. In der Nachfolge der großen memorialistischen Romane lässt unser Autor in diesen Episoden das kanonische Motivarsenal Revue passieren, mit dem der Krieg bereits von Henri Barbusse, Ernst Jünger,

21 Siehe zu ihrer Rezeption Faria 2013.

22 Eine vergleichbare Fallanalyse aus diesem Segment bietet Schwarze 2016.

23 Echenoz 2012. Der Text wird im Fließtext unter Angabe der Seitenzahlen in Klammern zitiert. 24 Eine hervorragende Einführung in die Poetik des Kriegsromans bietet Kaempfer 1998, zur „rhétorique“ der zeitgenössischen Produktion insbesondere 237-273. Siehe zu den Formen der romanesken Modellierungen des Krieges grundlegend auch Schoentjes 2009. 
Rainer-Maria Remarque, Roland Dorgelès, Maurice Genevoix und vielen anderen ausbuchstabiert worden war. So lesen wir bei Echenoz von der Mobilmachung im August 1914, unendlichen Zwangsmärschen, grausamen Gefechten, von Verstümmelungen, Tod und Fahnenflucht, von Kriegstribunalen und Exekutionen. Und auch das zivile Leben hinter der Front samt der Kriegswirtschaft, die Heimkehr der Veteranen und ihre prekäre sozio-ökonomische Lage sowie die Probleme bei ihrer Reintegration kommen hier zu Wort. Echenoz' Kriegserzählung wirkt in all dem prima facie wie eine konventionelle historische Fiktion, die das hinlänglich bekannte Wissen über die Grande Guerre ein weiteres Mal in komprimierter Form wiedererzählt.

Im Feuilleton hat dieser Eindruck Echenoz zum Teil harsche Kritik eingebracht. So verriss Andreas Isenschmid den Roman mit dem Argument, „auch wer noch kein Buch über den Ersten Weltkrieg gelesen“ habe, werde „aus Echenoz' Roman nicht erfahren, was er nicht schon längst gewusst hätte.“25 Diese am historiographischen Kenntnisstand orientierte Kritik geht freilich am Interesse von 14 vorbei, wie bereits augenfällige Einlassungen in den Roman zeigen, die ausdrücklich markieren, dass hier gerade nicht Neues über den Kriegsverlauf zutage gefördert werden soll. In diesem Sinne heißt es etwa am Ende des 10. Kapitels, das evoziert, welche Qualen der tagelange Artilleriebeschuss und die Gasangriffe für die Soldaten in den Schützengräben bedeuten: „Tout cela ayant été décrit mille fois, peut-être n'est-il pas la peine de s'attarder encore sur cet opéra sordide et puant.“ (79) ${ }^{26}$ Solche Erzählerkommentare vermitteln lakonisch, dass sich Echenoz' Roman an ein informiertes Publikum richtet, welches über ein gesichertes Wissen über den Ersten Weltkrieg verfügt und mit der literarischen Tradition der grands récits de la Grande Guerre vertraut ist. ${ }^{27} 14$ kann damit als eine historische Metafiktion postmodernen Zuschnitts charakterisiert werden, ${ }^{28}$ die Echenoz bewusst sowohl vor der Folie des historischen Diskurses über den Krieg als auch vor derjenigen möglicher Rezeptionshaltungen und -erwartungen einer vorgebil-

25 Isenschmid 2014, 58.

26 Zu Beginn des abschließenden 15. Kapitels erklärt die Erzählstimme analog dazu: „On connaît la suite." (115)

27 So auch Schoentjes 2014, 262, der betont, Echenoz setzte einen „lecteur créatif“ voraus, der in der Lage sei, die intertextuellen Anspielungen, thematischen Leerstellen und die fragmentarische Bildlichkeit zu vervollständigen. Dass Echenoz seinerseits die klassischen Kriegsromane genau kennt, belegen Äußerungen in Interviews, in denen er neben Augenzeugenberichten und historiographischen Darstellungen vor allem die Romane von Henri Barbusse, Gabriel Chevalier, Roland Dorgelès, Maurice Genevoix, Ernst Jünger und Erich Maria Remarque als seine vorbereitenden Lektüren nennt. www.leseditionsdeminuit.fr/livre-14-2758-1-1-0-1.html [19.11.2016].

28 Siehe dazu Nünning 1995. 
deten Leserschaft inszeniert. Sein Interesse, so können wir annehmen, besteht daher darin, den Krieg wiederzuerzählen, um ihn neu zu perspektivieren.

Dieser Anfangsbefund lenkt den Blick auf die Darstellungsweise des Romans, näherhin darauf, mit welchen Mitteln er die historische Erfahrung modelliert. Bevor ich dieser Frage für 14 nachgehe, gilt es, sie zunächst in methodologischer Hinsicht kurz zu beantworten. Ich rufe dazu zwei basale narratologische Kategorien auf, die das Verhältnis der Narration zu dem von ihr Dargestellten in jeder Form des Erzählens von der Wirklichkeit betreffen. Sie lassen sich einfach mit dem Begriffspaar Distanz und Nähe fassen. ${ }^{29}$ Seine Relevanz für die Modellierung von Wirklichkeit wird klar, wenn wir uns vor Augen führen, dass sich die Erzählung und zumal diejenige von der Vergangenheit grundsätzlich mit einem zweifachen referentiellen Anspruch konfrontiert sieht: Einerseits ist es die Aufgabe der narrativen Repräsentation von Wirklichkeit, die Komplexität historischer Umstände und Geschehnisse zu reduzieren, um der Vergangenheit eine kohärente Erklärungsstruktur zuschreiben zu können. Andererseits möchte gerade der Geschichtsroman der Komplexität des Vergangenen Eviden $z^{30}$ verleihen und evoziert $\mathrm{zu}$ diesem Zweck die Offenheit und Kontingenz vergangener Wirklichkeitswahrnehmungen. Man kann die referentielle Grundspannung, in der sich die Geschichtserzählung damit stets bewegt, einerseits als die Herstellung von explikativer Kohärenz bezeichnen und andererseits als die Ausstellung von Kontingenz. ${ }^{31}$ Der kategoriale Konnex von Kohärenzherstellung und Kontingenzexposition kann insofern als eine Grundannahme allen historischen Erzählens gelten. Diese Beobachtung legt es hinsichtlich der Qualität der historischen Referentialität von Geschichtsromanen nahe, zwischen zwei alternativen Möglichkeiten zu unterscheiden: Entweder die erzählte Geschichte

29 Siehe zum Modus der distance die nach wie vor grundlegende Konzeptionalisierung von Gérard Genette: „Discours du récit“, in: Genette 1972, $184 \mathrm{ff}$.

30 Der Begriff der Evidenz wird hier Ludwig Jäger folgend im Sinne einer Semantik verwendet, die im Zeichenmodus der „Transparenz“ funktioniert. Dies bedeutet, dass Evidenzeffekte die Verfahren zur semiotischen Herstellung ihrer unmittelbaren Wirkung im besten Fall kaschieren können. Siehe Jäger 2008, Zitat 310.

31 In einer systematischen Studie hat Rainer Warning (2001) das Verhältnis von „Kontingenzbewältigung und Kontingenzexposition“ bei einer Reihe moderner französischer Erzähler von Balzac, über Flaubert, Céline und Gide bis zu Ricardou und Claude Simon untersucht. Sein Interesse gilt dabei Erzählweisen, welche nicht linear progredieren, sondern Erfahrung von Kontingenz im Rahmen eines „spezifisch modernen, ateleologischen Zeitbewußtsein[s]“ (199) implizit zum Gegenstand erheben. Von diesem Phänomen, bei dem der Text „die referentielle Kontingenz immer und zugleich auch als kreative Kontingenz“ (206) exponiert und reflektiert, ist der hier behandelte Roman zu unterscheiden. Denn er beschränkt sich darauf, referentielle Kontingenz zu inszenieren, und erzählt damit bewusst im Syntagma. 
zielt darauf ab, unmittelbare Wahrnehmungen und individuelle oder kollektive Wirklichkeitserfahrungen $\mathrm{zu}$ evozieren und so ästhetisch nachvollziehbar $\mathrm{zu}$ machen, oder aber sie steht im Dienste der kausal-logischen Rekonstruktion historischer Zusammenhänge. Diese Oppositionsbildung transportiert die Hypothese eines Antagonismus zweier Modi von Referenzbildung, welche bis heute weit verbreitet ist. ${ }^{32}$ Die folgenden Überlegungen stellen eine solche strukturelle Gegensätzlichkeit zweier konkurrierender Modi des Referenzbezugs in Frage. Sie gehen vielmehr von der methodischen Grundannahme aus, dass sich das, was man verkürzt als das Erklären und das Zeigen von Geschichte bezeichnen könnte, grundsätzlich komplementär zueinander verhält.

Untersucht man im Bereich der Literatur zur Grande Guerre die Mittel, welche die Romane zu Modellierung der historischen Wirklichkeit einsetzen, lässt sich tendenziell eine typische, wenig überraschende Zuordnung beobachten. Sie besteht darin, dass textuelle Verfahren der Distanzierung vom Gegenstand häufig im Dienste einer explikativen Rekonstruktion der Vergangenheit stehen, während Näheeffekte häufig erzeugt werden, um die chaotische Komplexität vergangener Geschehnisse zu evozieren. Beispiele für vorrangig auf die Wirklichkeitsexplikation abzielende Darstellungen in der kriegsnahen Romanproduktion sind Pierre Chaines Mémoires d'un rat suivi des commentaires de Ferdinand, ancien rat de tranchées (1917), Georges Duhamels Civilisation 1914-1917 (1918) oder in den 1930er Jahren La Comédie de Charleroi (1934) von Drieu La Rochelle; die memorialistische Exposition von Kontingenz hingegen steht im Mittelpunkt von Le feu. Journal d'une escouade von Henri Barbusse (1916), in Les croix de boix (1919) von Roland Dorgelès oder Jean Gionos Le grand troupeau (1931) und anderen mehr. Unter den Gegenwartsromanen werden die genannten semantischen Zuordnungen von Distanz- bzw. Näheeffekten in ausgeprägter Weise zum Beispiel durch Erstlingsromane Le théorème de Roitelet von Frédéric Cathala (2004) respektive Cris (2001) von Laurent Gaudé repräsentiert. ${ }^{33}$

32 So etwa bei Gianfranco Rubino, der die nach 2000 erschienene französische Romanproduktion zum Ersten Weltkrieg folgendermaßen charakterisiert: „Deux modèles s'opposent : d'une part, une tentative de rendre compte du chaos en le maîtrisant par une sorte d'esprit de géométrie, de l'autre, la conscience de l'impossibilité de représenter l'absurde sinon par un langage qui lui ressemble, broyé, pulvérisé.“ (Rubino 2009, 134)

33 Siehe zu Cathala Theeten 2009, 161-206 („La Grande guerre à travers le prisme de l'ironie“), sowie zu Gaudé 207-240 („La Grande Guerre revisitée par l'écriture de l'insolite“). Theeten behandelt die beiden Romane und andere mehr als Beispiele für die literarische Dekonstruktion von Klischees über den Ersten Weltkrieg bzw. für dessen literarische Ontologisierung. 
3 Damit komme ich zur écriture des Krieges in 14. Echenoz erzählt den Plot mit sprachlich-narrativen Verfahren, welche die auf den ersten Blick realistisch erscheinende Darstellung gezielt derealisieren. Dies heißt, dass die ,Welt im Text“ durch den Modus des Erzählens permanent als eine konstruierte Welt gekennzeichnet wird. Der Text entzieht dem Erzählten auf diese Weise den Eindruck einer unmittelbaren semantischen Referentialität und er markiert fortlaufend, dass es sich bei 14 um eine im Vorfeld von 2014 und nicht etwa im Kontext von 1914 erdachte Fiktion handelt. ${ }^{34}$ Die für die (post)moderne Literatur typische Durchkreuzung der Illusion des Fiktiven basiert bei Echenoz grundsätzlich auf einer écriture minimaliste. ${ }^{35}$ Die Derealisierung nämlich manifestiert sich unter anderem darin, dass das Dargestellte extrem verdichtet und kondensiert wird, was sich vorderhand in der Kürze des Romans niederschlägt. Diese Kürze basiert ihrerseits darauf, dass wir es bei 14 im Vergleich zur realistischen Tradition mit einer stark reduzierten Syntax zu tun haben, die komplexe hypotaktische Perioden durch parataktisch nominalisierende Konstruktionen ersetzt. Dem korrespondiert eine umso größere Sorgfalt im Lexikon und eine Darstellungsweise, die die Geschichte vielfach weniger in ihrer zeitlichen Entwicklung erzählt, als dass sie Dinge beschreibt, benennt und aufzählt.

Ein textuelles Phänomen, das im Rahmen dieser komprimierenden Oberflächenrepräsentation in 14 ins Auge fällt, sind lange, katalogartige Aufzählungen, die den Text durchsetzen. Auffallend ist an ihnen zunächst die Tatsache, dass sie verhältnismäßig viel Raum einnehmen. Ein aufschlussreiches Beispiel dafür findet sich in Kapitel 6, das von den kraftraubenden Tagesmärschen erzählt, die der Protagonist Anthime und die drei Kumpel, die mit ihm in die Infanterie eingezogen worden sind, in der Phase der großen Truppenbewegungen durchzustehen haben. Der Text beschreibt dabei ausführlich die Tornister, die die Rekruten tragen müssen, und erstellt dazu auf vollen zwei Seiten (48-50) ein detailliertes Inventar des Marschgepäcks. ${ }^{36}$ Die Menge an Gegenständen, die hier aufgelistet werden, schafft für die Vielzahl von Gegenständen, die jeder der jungen Män-

34 Der allusive Titel 14 zitiert offensichtlich Maurice Genevoix’ Ceux de 14 und eröffnet damit eine zeitliche Spannung, die auf die beinahe hundertjährige Geschichte literarischer Modellierungen des Weltkriegs verweist.

35 Siehe zu den Charakteristika des Minimalismus, die Echenoz’ Erzählungen generell kennzeichnen, Sermier 2013.

36 Die Regelausrüstung der Rucksäcke zum Beispiel umfasst „en matériel alimentaire - bouteilles d'alcool de menthe et substitut de café, boîtes et sachets de sucre et de chocolat, bidons et couverts en fer étamé, quart en fer embouti, ouvre-boîte et canif -, en vêtements - caleçons court et long, mouchoirs en coton, chemises de flanelle, bretelles et bandes molletières -, en produits d'entretien et de nettoyage - brosses à habits, à chaussures et pour les armes, boîtes de graisse, de cirage, de boutons et de lacets de rechange, trousse de couture et ciseaux à bouts ronds -, en effets 
ner auf langen Tagesmärschen zu schultern hat, gewissermaßen ein quantitatives Äquivalent auf der Textseite. Indem Echenoz die Belastungen der Soldaten in solchen minutiösen Auflistungen gewissermaßen inventarisiert, zielt er offensichtlich darauf ab, die materielle Dimension der Kriegserfahrung möglichst präzise abzubilden. ${ }^{37}$ Der Effekt der Präzision beruht dabei auf einer deskriptiven Dichte, welche die alltägliche Abhängigkeit der Rekruten von den Ausrüstungsgegenständen des Krieges seriell vermittelt. Die enumerativen Beschreibungen der Dingwelt stellen auf diese Weise den Eindruck referentieller Nähe her.

Es geht damit jedoch zugleich eine Distanznahme einher, die sekundärer Art ist und metafiktional funktioniert: Sie betrifft die intertextuelle bzw. mediale Ebene seiner Kriegsdarstellung und manifestiert sich im zitierten Beispiel darin, dass die Tornister-Sequenz in 14 als ein distantes Echo einer thematisch analogen Episode in Le feu von Henri Barbusse gelesen werden kann. Der epochale Kriegsroman von 1916 erzählt bereits das Schicksal einer Gruppe von jungen Soldaten, die zu einer Korporalschaft gehören, und schildert im fünften Kapitel einen Fußmarsch von 28 Kilometern. Barbusse thematisiert dabei ebenfalls ausführlich die großen Belastungen der Soldaten. ${ }^{38}$ Er schildert diesen „unendlichen“ Marsch mit der ihm eigenen naturalisierenden Expressivität als lange mechanische Bewegung einer Masse. Er setzt dabei auf den Effekt der Empathie, die das Leiden seiner Einheit gleichsam zu einem kollektiven Opfergang zwischen Hoffen und Bangen werden lässt. Gezielt anders erzählt Echenoz 2012: An die Stelle der episierenden Modellierung der Truppenbewegungen bei Barbusse setzt er eine Darstellungsweise, die das alles überhöhende Pathos kassiert, indem sie das Leiden gleichsam in aufzählenden Katalogen vergegenständlicht und auf seine Materialität reduziert. Echenoz markiert so mittels der réécriture eines Topos der Kriegsliteratur die zeitliche und ästhetische Distanz zu einem seiner klassischen Prätexte, welcher den Krieg als die Geburtsstunde einer Schicksalsgemeinschaft interpretiert und dazu eine Art Schlachtengemälde ausmalt.

de toilette et de santé - pansements individuels et coton hydrophile, torchon-serviette, miroir, savon, rasoir avec son aiguisoir, blaireau, brosse à dents, peigne - ainsi qu'en objets personnels tabac et papier à rouler, allumettes et briquet, lampe de poche, bracelet d'identité à plaques en maillechort et aluminium, petit paroissien du soldat, livret individuel.“(48-49) Echenoz evoziert das gleichsam erdrückende Gewicht der Tornister, um abschließend lakonisch anzudeuten, was es für die Träger bedeutete, wenn sie vom Regen vollgesogen waren: „L'ensemble de cet édifice avoisinerait alors au moins trente-cinq kilos par temps sec. Avant qu'il ne se mette, donc, à pleuvoir.“ (50)

37 Metatextuell wird dies ausgestellt, indem alleine in Kap. 6 dreimal die beinahe wortgleiche Formulierung „les choses se précisaient“ wiederholt wird. Vgl. 42, 45, 46.

38 Barbusse 2011, 75-102. 
Eine ähnliche intertextuelle Distanzmarkierung findet sich signalhaft bereits im ersten Kapitel des Romans, in dem Echenoz die Mobilmachung vom 1. August 1914 vor der Kontrastfolie einer glorifizierenden Szene in Victor Hugos TerreurRoman Quatre-vingt-treize von 1874 inszeniert. ${ }^{39}$ Echenoz zitiert dazu aus Hugos „dickem Buch“ explizit ein Kapitel, in dem der Held, von hoffnungsvoller Ahnung getragen, das Läuten der Alarmglocken anlässlich des Vendée-Aufstands von 1793 als ein erhebendes auditives Erlebnis wahrnimmt. Der Romanbeginn von 14 retextualisiert diese Szene, kehrt sie jedoch durch die Wahrnehmungsperspektive eines überaus gewöhnlichen Mannes um, dem jeglicher Heroismus fremd ist. So wird Anthimes eigentlich lebensrettende Leistung schlicht darin bestehen, sich an Krieg anzupassen. ${ }^{40}$

Neben dieser Art von intertextueller Distanzierung thematisiert Echenoz in 14 in analoger Absicht auch konventionelle bildliche Repräsentationsformen des Krieges. Dabei zitiert er kollektive Vorstellungen, die das mediale Gedächtnis der Grande Guerre bevölkern in der Absicht, ihre epischen Darstellungsweisen von den ,Nahaufnahmen' seiner Fiktion abzusetzen. ${ }^{41}$ Echenoz' Repräsentant solch einer erinnerungskulturell stark wirksamen, panoramatisch-heroisierenden Ikonographie des Krieges ist Anthimes hochmütiger Bruder Charles: ${ }^{42}$ Ausgerüstet mit einer Kamera, die bezeichnenderweise den Namen „Rêve Idéal“ (13) trägt, macht der erfolgreiche Presse- und Militärphotograph mit „unbeteiligtem Blick“ (13) gestellte Aufnahmen von Offizieren und photographiert im Dienste der Luftaufklärung vom Flugzeug aus weit entfernte Landschaften. Diesen selbstsicheren Solitär lässt Echenoz im siebten Kapitel (51-57) auf tragisch-komische Weise bei einem Flugzeuggefecht sterben. Der Text verabschiedet mit diesem Tod zugleich zeichenhaft einen visuellen Repräsentationsmodus, der hier offensichtlich

39 Echenoz lässt seinen Protagonisten auf einem Sommerausflug ein „gros livre“ lesen, das für sein Fahrrad viel zu schwer ist (11). Bei einem starken Windstoß, „le gros livre est tombé du vélo, s'est ouvert dans la chute pour se retrouver à jamais seul au bord du chemin, reposant à plat ventre sur l'un de ses chapitres intitulé Aures habeat, et non audiet.“ (11) Vgl. Hugo 1976, chap. IV, 2, 110-113. Siehe für eine detailliert intertextuelle Lektüre dieser Sequenz Oster-Stierle 2016.

40 Diesen Umstand betont der Text wiederholt, vgl. 70, 84, 107. Die Inszenierung eines solchen passiven und ,entpolitisierten“ Protagonisten und anderer „personnages dévitalisés“, die auf die Erfahrung des Krieges nicht im Modus der „Revolte“ reagieren, hat den engagierten Protest eines Teils der Kritik hervorgerufen. Siehe insbesondere die auf Clément Rosset aufsetzende Lektüre von Chaudier 2014.

41 So zum Beispiel, wenn der Text bei der Beschreibung des Aufbruchs der Soldaten en passant ein Monumentalfresko von Albert Herter aufruft, das den Titel Le départ des poilus, août 1914 (1926) trägt und heute in der Gare de l'Est zu sehen ist (vgl. Echenoz 2012, 21). Siehe zu den Techniken der Visualisierung in 14 Schoentjes 2014.

42 Vgl. Charles' Portrait, 16f. 
als negative Folie für die textuelle Nahperspektive der Echenoz'schen Darstellungsweise bzw. die Wirklichkeitswahrnehmung seines Protagonisten Anthime fungiert.

Die bis hierher skizzierte Gleichzeitigkeit, die Echenoz zwischen referentieller Nähe und intertextueller bzw. medialer Distanz herstellt, ist für die Modellierung des Krieges von Bedeutung, weil unser Text auf diese Weise tradierte gemeinschaftsstiftende Sinnzuschreibungen für den Ersten Weltkrieg verneint. Dies wirft die Frage auf, was in ihm an die Stelle solch letztlich (selbst)legitimierender Attribuierungen tritt. Anders gefragt: Haben wir es einfach mit einer typisch postmodernen historischen Metafiktion zu tun, die den Krieg im Modus der Uneigentlichkeit retextualisiert, oder verfolgt Echenoz auch einen referentiellen Zweck damit, dass er in den Aufzählungen die besagte Nähe zur Materialität des Kriegsalltags erzeugt? Im Gegensatz zur Überzahl der Kritiken bin ich der Meinung, dass letzteres der Fall ist, Echenoz den Krieg also auch wiedererzählt, um durchaus historisches Wissen $\mathrm{zu}$ vermitteln. ${ }^{43}$

Nach dem, was eingangs über die Zuordnung von Nähe- bzw. Distanzeffekten zur Ausstellung von Kontingenz respektive zur Herstellung von explikativer Kohärenz gesagt wurde, liegt es nahe, in den Listen vor allem ein Mittel zu sehen, das darauf abzielt, die unmittelbare Wahrnehmung des Krieges zu veranschaulichen. Dies ist in der Tat eine Seite der Medaille. Die andere jedoch ist - und darauf kommt es mir in dem Punkt an -, dass die Erzeugung von Nähe zur Objektwelt in 14 zugleich dazu dient, einer Dimension dieses Krieges thematisch Profil zu verleihen, die in der literarischen Tradition meist anderweitig überformt wird. Diese Dimension betrifft den Gebrauchswert, den Ausrüstungsgegenstände und insbesondere technologische Innovationen im Kriegsalltag für die Soldaten haben. In diesem Sinne beschreibt Echenoz minimalistisch, von welchem praktischen Nutzen Schuhe, Schutzhelme, Waffen, Flugzeuge oder eben die vollkommen überladenen Marschtornister für diejenigen sind, die auf diese Gerätschaften angewiesen sind. Um ihren faktischen Gebrauch zu erklären und die Frage ihrer Funktionalität zu bewerten, bedient sich die Erzählstimme dabei immer wieder inkongruenter Vergleiche, Metaphern und Metonymien.

Ein Beispiel für diese gezielt dissonante Verwendung von Tropen findet sich im 12. Kapitel, das mit „Les animaux dans la guerre“ überschrieben sein könnte. Es listet im Wesentlichen die Tiere auf, mit denen die Soldaten an der ländlichen Front zusammenleben, und klassifiziert sie absteigend nach dem Nutzwert

43 Die Frage der Referentialität des Dargestellten stellen sich weder Schoentjes 2012 und 2014 noch Mikowski 2015. Oster-Stierle 2016 geht sogar so weit, die Möglichkeit, dass es dem Roman auch um eine adäquate Vermittlung von historischen Wissen gehen könne, kategorisch zu verneinen: „Il ne cherche pas à transmettre un savoir historique.“ (138) 
bzw. der Schädlichkeit, welche die Tiere für die prekäre Ernährungslage der poilus haben. Dieser sechsseitige catalogue raisonnée (87-93) bildet Tier-Klassen und charakterisiert sie in bewusst anachronistischer Weise nach sozio-ökonomischen Kategorien von Nützlichkeit, die heute gängige Verwaltungstermini aufrufen. Da ist von herrenlosen „Nutztieren“ (,animaux utilitaires“; 87) die Rede, zu denen die „poulets et coqs en voie de marginalisation“ oder die Hasen „sans domicile fixe“ (88) zählen. Während diese Tiere sich gut verzehren lassen, kommen Haustiere, die „plus familiers, domestiques voire décoratifs“ (89) sind, und solche, die „purement ornamentaux“ (89) daherkommen, dafür kaum in Frage. Zu dieser Kategorie zählt der Erzähler Hunde, Katzen und Käfigvögel. Des Weiteren werden die „animaux indépendants“ (90) wie Rehe oder Wildschweine verzeichnet, die den Status von „franc-tireurs“ haben und keinem „code de travail“ (90) unterliegen. Schließlich folgt eine lange Beschreibung der „marginaux“ (91): Es ist dies „toute sorte de parasites irréductibles“(92), die „aucun appoint nutritionnel“ (92) bietet, sondern - „comme des monosyllabes“ (93) - ein einziges Ziel verfolgt: seinen eigenen Hunger „voracement“ an der Truppe zu stillen. Neben den Wanzen, Zecken, Mücken und Bremsen ist dies vor allem das Heer fresslustiger und fortpflanzungsfreudiger Flöhe, Läuse und Ratten.

Das ironische Bild, das dieser kommentierte Katalog vom Verhältnis zwischen Menschen und Tieren an der Front zeichnet, relativiert drastisch mögliche Vorstellungen davon, die Tiere seien „mascotte[s] de compagnie“ (90) oder treue Gefährten der Soldaten gewesen. ${ }^{44}$ Hier werden sie vielmehr auf ihren Wert als eine rare, existenzsichernde Nahrungsquelle reduziert und in der Mehrzahl als „potentiellement guerriers“ (92) vorgeführt. Pointiert formuliert der Erzähler diese Kernaussage zum Abschluss der Aufzählung, wenn er die „Parasiten“, neben dem militärischen Gegner und der eigenen Feldpolizei, lakonisch zum dritten Hauptfeind in einer für die Soldaten so gut wie ausweglosen Lage erklärt:

Or on ne quitte pas cette guerre comme ça. La situation est simple, on est coincés : les ennemis devant vous, les rats et les poux avec vous et, derrière vous, les gendarmes. (94)

Die gesamte Sequenz wie auch diejenige über die Tornister und andere mehr zeigen, dass Echenoz' derealisierende Aufzählungen und Beschreibungen konsequent Nähe zu ihren Gegenständen erzeugen, nicht zuletzt um mittels dieser stark komprimierenden Erzählweise etwas über die historische Wirklichkeit des Krieges jenseits des Textes auszusagen. Der referentielle Aussagewert der Fiktion besteht dabei darin, die Kriegserfahrung der Soldaten indirekt über die Inszenierung des Verhältnisses zu charakterisieren, in dem sie zur materiellen Welt

44 Siehe dazu in historischer Perspektive Wischermann 2015. 
stehen, oder zur Fauna wie im letzten Beispiel. Dabei rückt insbesondere der syntaktische Minimalismus der Aufzählung die Materialität des Krieges in das Zentrum der Darstellung und eliminiert auf semantischer Ebene zugleich mögliche politische, ideologische, ästhetische oder psychologische Überhöhungen. Man könnte diese Konzentration auf die materiellen Beziehungen im Krieg als materielle Gebrauchsgeschichte bezeichnen. ${ }^{45}$

Im Rahmen der literarischen Repräsentation der Grande Guerre fokussiert 14 damit einen Aspekt der Fronterfahrung, der zum einen in zeitgenössischen Schriftquellen wie Feldpostbriefen oder Tagebüchern einen wichtigen Platz einnimmt ${ }^{46}$ und der zum anderen in den letzten Jahren auch von Teilen der Geschichtsschreibung vermehrt ins Visier genommen worden ist. Dieses historiographische Interesse nähert sich, so mein Eindruck, dem Untersuchungsgegenstand auf einer Mikroebene und gilt den sozialen Nahbeziehungen aller Akteure im Krieg. Stellvertretend für diese Tendenz sei der Historiker Stéphane Audoin-Rouzeau genannt; in seinen Studien verfolgt er die Rekonstruktion einer „histoire intégrée“ „à hauteur des hommes“, welche jegliche Form zeitgenössischer Praktiken und Repräsentationsweisen des Ersten Weltkriegs umfasst. ${ }^{47}$ Audoin-Rouzeau, der unter anderem als Mitherausgeber einer grundlegenden Encyclopédie de la Grande Guerre fungiert, ist den mentalitätsgeschichtlichen Ansätzen der Annales-Geschichtsschreibung verpflichtet und rekurriert nicht zuletzt auf private Schriftquellen, wie sie inzwischen die Internetseiten des Projekts Europeana Collection 1914-1918 tausendfach digital zur Verfügung stellen. ${ }^{48}$ Es wäre gewagt, zwischen solchen Ansätzen der Geschichtswissenschaft und dem Roman von Echenoz eine direkte Verbindung herstellen zu wollen. Das

45 Bestätigung findet diese Beobachtung ante litteram implizit in einem Interview, das Jean Echenoz 2009 nach Erscheinen seiner biographischen Erzählungen Ravel (2006) und Courir (2008) der Zeitschrift Siècle 21 gab. Darin erklärt der Autor hinsichtlich seines literarischen Interesses an Menschen: „La psychologie, du côté de mon travail, ne m'intéresse pas : je n'ai jamais voulu entrer dans les états d'âme des personnages mais essayer plutôt d'en faire déduire les éléments par leurs comportements, leur rapport matériel au monde dans une ligne, disons béhavioriste, dont le modèle est Dashiell Hammett.“ Echenoz 2010, 121.

46 Es ist in diesem Zusammenhang signifikant, dass Echenoz in einem Interview angab, der Anlass des Romans seien die Kriegstagebücher eines einfachen Soldaten gewesen, die er in einem Familiennachlass gefunden und zunächst transkribiert habe. Vgl. www.leseditionsdeminuit.fr/ livre-14-2758-1-1-0-1.html [19.11. 2016]. Vgl. dazu mit Blick auf die Kriegstagebücher von Ernst Jünger und Carlo Emilio Gadda Schwarze [im Druck].

47 So Audoin-Rouzeau und Becker in der „Introduction“ zu der von ihnen herausgegebenen Encyclopédie de la Grande Guerre (Audoin-Rouzeau/Becker 22012, 7-14, Zitat 12). Vgl. auch AudoinRouzeau 2008.

48 Siehe hierzu www.europeana-collections-1914-1918.eu/ [11.11.2016]. 
vergleichbare referentielle Interesse beider Wirklichkeitsdiskurse an der revisitation des Krieges scheint jedoch tendenziell darauf hinzudeuten, dass sich seit einigen Jahren in Teilen der Geschichtswissenschaft eine Besinnung auf das Erzählen vollzieht. ${ }^{49}$ Erwähnt sei hier lediglich der schwedische Historiker Peter Englund, der den Ersten Weltkrieg jüngst originell perspektiviert hat, indem er auf der Basis von Schriftquellen die „Schicksale“ von 19 Personen im Krieg in ebenso vielen parallelen Erzählsträngen präsentiert. ${ }^{50} \mathrm{Ob}$ sich aus dieser Anfangsbeobachtung eine weiterreichende Tendenz zur narrativen Annäherung von Zeitgeschichtsschreibung und literarischem Erzählen vom Krieg ablesen lässt, ist eine komplexe Frage, die meines Erachtens weitere Beachtung verdient.

Festzuhalten bleibt hier als Zwischenfazit, dass Jean Echenoz in 14 insbesondere das Banale, das Alltägliche, das „Infra-Ordinäre“51 in langen Listen detailliert inventarisiert, um eine materielle Gebrauchsgeschichte des Kriegs zu erzählen. Er wendet damit gezielt ein de-realisierendes Erzählverfahren an, das gleichwohl einen referentiellen Aussagewert besitzt, indem es über die Erzeugung von textueller Nähe zu den Gegenständen der Rede dazu beiträgt, das Wissen über den Krieg im Sinne kohärenter Erklärungen literarisch auszudifferenzieren.

Der letzte Schritt dieser Analyse behandelt die Frage, wie Echenoz im Rahmen seiner écriture minimaliste Verfahren narrativer Distanzierung einsetzt. Das wichtigste Mittel hierfür ist, wie die wenigen Textbeispiele bereits deutlich gemacht haben, eine lässige Ironie, die das, was sie ironisch bricht, nicht verurteilt, sondern mit einem lakonischen Lächeln belegt. In 14 manifestiert sich diese Ironie in den bereits erwähnten dissonanten Tropen. Zum Ausdruck kommt sie zudem in entpersönlichenden, gleichsam technischen Beschreibungen grausamer Szenen von Verletzung und $\operatorname{Tod}^{52}$ sowie drittens in permanenten Registerwechseln, die einen Kontrast zwischen einem preziös-elaborierten code écrit und salopp-

49 Im Fall von Audoin-Rouzeau ist dies offensichtlich, wenn man zudem bedenkt, dass der Historiker jüngst mit Quelle historie. Un récit de filiation (1914-2014) (Audoin-Rouzeau 2013) die Kriegsgeschichte seiner Familie in einer literarisierten Erzählung veröffentlicht hat.

50 Englund 2011.

51 Ich übernehme den Begriff von Georges Perec, der ihn in Abgrenzung zum traditionellen Erzählen des Extraordinären verwendet. Seine Poetik der „détails minisculs“ sucht die lebensweltliche Realität in der Notierung im „Banalen, Alltäglichen, Evidenten, Gemeinen, InfraOrdinären“, das den „bruit de fond“ der Existenz zugänglich macht. Vgl. „Approches de quoi ?“, in: Perec 1989, 9-13, Zitat 11. Siehe zur Bedeutung des Konzepts für Echenoz auch Sermier 2013, 55.

52 Ein Beispiel für mechanistisch-klinische Beschreibungen einer Gewaltszene stellt in Kapitel 11, 82f., die Gefechtsszene dar, bei der Anthime durch einen Querschläger seinen rechten Arm verliert. Ironisch wird die Darstellung dadurch, dass der bloße Zufall, der die Verletzung hervorruft, hier als hinterlistiges Attentat eines heimtückischen Granatsplitters dargestellt wird. Den 
umgangssprachlichen Ausdrucksweisen des langage parlé erzeugen. ${ }^{53}$ Diese Ironiemarker begründen in dem Text eine ausgesprochen lakonische Erzählweise, die das existentiell Dramatische bewusst mit lässiger Nonchalance behandelt. Der Zweck, den der Roman damit verfolgt, besteht darin, distanziert den Hiat zu thematisieren, der für die Soldaten zwischen der unmittelbaren Wahrnehmung existentiell bedrohlicher Situationen besteht und der Unmöglichkeit, individuell situationsgerecht darauf zu reagieren. Diese Form von Kontingenzerfahrung führt auf der Handlungsebene dazu, dass die Protagonisten akute Gefahren als unwirklich erleben und sich in einer Weise verhalten, die von außen betrachtet leicht als unangemessen, skurril oder schlicht absurd betrachtet werden kann. Die ironische Distanzierung der Rede von ihrem Gegenstand fungiert hier also als modaler Garant für die Exposition von Kontingenz.

Bevor ich als Beleg für diese Art von Kontingenzmodellierung kurz auf das achte Kapitel eingehe, in dem Anthime erstmals im Kampf Kontakt mit dem Feind hat, sei daran erinnert, dass es nichts Neues ist, dass der moderne Roman das komische Potential einer als irreal erlebten Realität des Krieges ausschöpft: Prototypisch steht dafür Stendhals La Chartreuse de Parme von 1839. Im dritten Kapitel des Romans wird Fabrice del Dongo, der junge Protagonist, inmitten der Schlacht von Waterloo ironisch als ein blauäugiger Soldat vorgeführt, der in eine ekstatische Verzückung versunken ist und dem daher die Kugeln um die Ohren fliegen, ohne dass er irgendetwas von dem, was vor sich geht, begreifen würde. ${ }^{54}$ Eine Wiederaufnahme dieses an die Figur des picaro erinnernden Kriegsteilnehmers stellt die ebenfalls dezidiert ironische, derealisierende écriture dar, mit der Jean Cocteau 1923 in Thomas l'imposteur seinen Protagonisten die Wirren hinter der Front meistern lässt. ${ }^{55}$ Und 1932 inszeniert Louis-Ferdinand Céline in Voyage au bout de la nuit, wie sein alter ego Bradamu die Front des Ersten Weltkriegs erlebt. Auch hier nimmt die Figur das Gefecht als etwas Unwirkliches wahr, auf das der Ich-

intertextuellen Dialog, den Echenoz hier augenscheinlich mit Blaise Cendras' Roman La main coupé (1918/1946) führt, behandelt Oster-Stierle 2016, hier 138ff.

53 Ein Beispiel hierfür ist das Gespräch zwischen Blanche und dem docteur Monteil, in dem beide die Möglichkeit einer Abtreibung besprechen. Die für die junge Frau dramatische Lage wird dabei ironisch durch das betont konversationelle Register eines Dialogs depotenziert, der als Fließtext ohne Interpunktion daherkommt, der von umgangssprachliche Ellipsen durchsetzt ist und in dem der Gebrauch von Pronomen dominiert, denen eine eindeutige Zuordnung zu Nomina fehlt. Siehe 14, 39f. Siehe zum Prinzip der Registerwechsel, die bei Echenoz vornehmlich dazu dienen, die materiellen Aspekte beschwerlicher Erfahrungen ironisch zu modellieren, Bouchy 2012.

54 Siehe dazu „L'école de l’ironie“, in: Pavel 2003, 260-268.

55 Vgl. Rehage 2001 sowie Adinolfi 2014, hier insbesondere 33ff. 
Erzähler im Nachhinein mit aggressiver Ironie zurückblickt. ${ }^{56}$ Indem Echenoz in 14 derartige Szenen variierend wiedererzählt, schreibt er die moderne Traditionslinie des desillusionierenden Kriegsromans fort; er erzeugt damit intertextuelle Nähe.

Sein Text praktiziert demnach die Reinszenierung einer Kontingenzerfahrung, die für den Kriegsroman als topisch gelten kann und die referentiell betrachtet keinen nennenswerten Mehrwert besitzt. Der Modus lakonischer Ironie jedoch, in dem Echenoz die Motivik neu erzählt, verfolgt ein anderes Ziel: Implizit, aber durchaus gezielt thematisiert sie die Pragmatik des Texts, näherhin die Frage des adäquaten Umgangs mit dieser Art von Erfahrung. Gemeint sind Erwartungshaltungen, mit denen die Leserinnen und Leser seiner Reinszenierung des Kriegs begegnen mögen. Wie Echenoz diese pragmatische Ebene der Rezeption in seine Narration einbezieht, sei kurz anhand des Beginns von Kapitel 8 gezeigt. Anthime steht hier zum ersten Mal im Feuer und der Text transportiert die unbekümmerte Kopflosigkeit, mit der er diesen dramatischen Moment erlebt, im Modus der Ironie. Das Handlungsvakuum, das dabei auf Seiten des Protagonisten besteht, verdichtet der folgende Satz, der die Situation auf den Punkt bringt:

Puis on leur a crié d'avancer et, plus ou moins poussé par les autres, il [Anthime, M. S.] s'est trouvé sans trop savoir que faire au milieu d'un champ de bataille on ne peut plus réel. (59)

Zum Ausdruck kommt hier der Gegensatz zwischen dem anonym bleibenden Befehl zum Vormarsch und der damit verbundenen Aufforderung zum Kampf einerseits und der Orientierungslosigkeit Anthimes andererseits. Eine wichtige Rolle spielt dabei die Verwendung der Personalpronomina: Die Figuren, allen voran Anthime, werden von der extradiegetischen Erzählstimme wechselweise entweder mit der 3. Ps.Sg. [,il'] bzw. ihrem Namen bezeichnet oder sie werden einem kollektiven ,on' subsumiert. Dies bedeutet, dass sie mal persönlich identifiziert werden, mal als Teil einer entpersönlichten Gemeinschaft auftreten. Das Pronomen ,on' repräsentiert dabei meist undifferenziert die anonyme Gesamtheit der Infanterie, einschließlich der gesichtslosen Heeresführung, deren Entscheidungen und Handlungen für die poilus opak bleiben. Die Mehrdeutigkeit des Pronomens ,on' nutzt der Text hier, um die Diskrepanz offenzulegen, die zwischen dem individuellen ,il‘ und einem entpersönlichen ,on' hinsichtlich der kognitiven und emotionalen Wahrnehmung und Verarbeitung der nun eingetretenen Gefechtssituation besteht. Verschärft wird dieser Kontrast durch das umgangssprachliche Syntagma „un champ de bataille on ne peut plus réel“, das ironisch wirkt, weil es einen diskursiven Kontrast zum vorherrschenden Register

56 Vgl. Quinn 2003. 
des code écrit und damit zugleich eine semantische Distanz zum existentiellen Ernst der Lage herstellt.. ${ }^{57}$ Die Bemerkung, man könne sich das Schlachtfeld, auf dem Anthime steht, echter nicht vorstellen, signalisiert so emphatisch, dass eigentlich akut höchste Handlungsbereitschaft gegeben wäre.

Die Verwendung der feststehenden Wendung ,on ne peut plus ‘ + Adjektiv bezieht sich hier jedoch nicht nur auf die histoire, sondern impliziert grammatisch auch die Ebene der Rezipienten. Dies liegt daran, dass das Personalpronomen ,on bekanntlich nicht nur dem unpersönlichen ,man' der 3. Pers. Sg. entspricht, sondern dass es genauso das identifizierbare ,nous' der ersten Person Plural bezeichnet; in der Umgangssprache ist dies der Regelfall. Im Zusammenspiel mit dem präsentischen Gebrauch des Verbs ,pouvoir' verweist die Formel ,on ne peut plus réel' daher auch auf die Gemeinschaft des Erzählers mit der potentiellen Leserschaft des Romans. Dies hat zur Folge, dass die ironische Erzählhaltung der Passage die Erwartungen der Leser einschließt. Der ironische Superlativ, das Schlachtfeld sei „so real wie nur möglich“, konfrontiert auf diese Weise nicht zuletzt uns Leser mit unseren eigenen, medialisierten Erwartungen an das Genre des récit de guerre. Kann es nicht sein, dass etwa die Vorstellung, in einer akuten Kampfsituation seien Soldaten kampfbereit und handelten situationsgerecht, eine naive Kohärenzerwartung ist, die nicht nur nicht der Kriegswirklichkeit entspricht, sondern die fundamental mit der kohärenzbildenden Funktion allen Erzählens an sich zu tun hat? Echenoz expliziert diese Zusammenhänge nicht. Doch dürfte deutlich geworden sein, wie der Distanzmodus der Ironie in 14 mehrere Bezugsebenen miteinander verschränkt, die das Thema eines kontingenten Handlungsvakuums betreffen: Intertextuell steht Echenoz' Modellierung des Phänomens in unmittelbarer Nähe zur Tradition des modernen Kriegsromans; referentiell stellt sie ebenfalls Nähe her, indem sie das besagte Erleben der Kontingenz reinszeniert. Schließlich durchkreuzt die Ironie potentiell naive Rezeptionshaltungen und erzeugt so metatextuell Distanz.

Am Beginn meiner Überlegungen stand die Frage nach der Referentialität der Geschichtsfiktion und die Absicht, entsprechende Textualisierungsmuster zu identifizieren, die Distanz- und Näheverhältnisse modellieren. Die Analyse des Kriegsromans von Jean Echenoz hat gezeigt, dass die Herstellung solcher Effekte vor allem zwei Bezugsebenen aufweist. Zum einen betrifft der metatextuelle Dialog mit konventionellen Repräsentations- und Rezeptionsweisen der

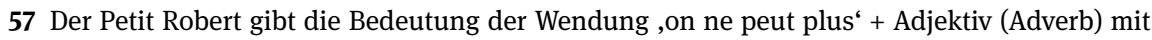
,au plus haut point (devant l'adj. ou l'adv.) $\rightarrow$ extrêmement‘ an. (Le nouveau Petit Robert de la langue française 2009. Version 3.2. (c) 2008 Dictionnaires LeRobert/Sejer) Das Syntagma drückt demnach den höchsten Grad einer gegebenen Qualität aus und könnte hier mit „absolument réel“ paraphrasiert werden. 
Grande Guerre, wie sie die Erzähltradition und das ikonographische Archiv dem kollektiven Gedächtnis zur Verfügung stellen, potentielle Leserinnen und Leser des Romans. Denn indem der Roman solche Darstellungsweisen des Krieges dezent auf Distanz bringt und dabei die Falle einer pathetischen Verharmlosung der Schrecken und Traumata dieses Krieges umgeht, ${ }^{58}$ grenzt er sich nicht nur kritisch von vielen zeitgenössischen Populärromanen über den Krieg ab, sondern konfrontiert seine Leserschaft zugleich kritisch mit lange eingeübten, möglicherweise unreflektierten Rezeptionserwartungen.

Zum anderen beziehen sich Distanz- und Näherelationen in 14 tatsächlich referentiell auf die historische Vergangenheit, von der der Roman primär handelt. Darum ging es hier in erster Linie. Echenoz' minimalistische Schreibweise lässt in diesem Punkt eine signifikante Inversion konventioneller Zuordnungen erkennen: Häufig dienen Verfahren, die sprachliche Effekte von Nähe und Unmittelbarkeit erzeugen, dazu, die Kontingenz der historischen Vergangenheit zu evozieren. Die Herstellung von narrativer Distanz hingegen steht vorzugsweise im Dienste einer rationalisierenden Erklärung und Systematisierung von Vergangenem. Echenoz kehrt dieses Verhältnis partiell um, denn die Konkretheit einer aus der Nähe registrierten Objektwelt hat bei ihm vornehmlich eine kohärenzstiftende und historisch erklärende Aufgabe. Sie trägt zur Ausdifferenzierung des Krieges im Sinne der Gebrauchsgeschichte seiner Materialität bei. Im Gegenzug stellt der Text zum Dargestellten vielfach eine ironische Distanz her, um einer zentralen Form von Kontingenzerfahrung im Krieg Ausdruck zu verleihen, der tiefen Kluft nämlich zwischen der unmittelbaren Wahrnehmung einerseits und andererseits der Unmöglichkeit, das Wahrgenommene kognitiv-emotional zu erfassen und in ein situationsadäquates Handeln zu überführen. Das Prinzip der narrativen Nähe korrespondiert damit bei Echenoz nicht zuletzt mit der Herstellung von historischer Kohärenz, während das Prinzip narrativer Distanzierung umgekehrt vermehrt im Dienste der Inszenierung von Kontingenz steht. 14 kann damit als ein Beispiel dafür gelten, wie narrative Effekte von Distanz und von Nähe in einer Geschichtsfiktion als komplementäre Referenzstrategien eingesetzt werden, die beide dem Zweck dienen, die simultanen Ansprüche der Geschichtserzählung einzulösen: verlebendigende Evokation und abstrahierende Rekonstruktion des Vergangenen zu sein.

Nimmt man die beiden Ergebnisse der Analyse zusammen, ergibt sich für Echenoz' Kriegsroman in der Zusammenschau das Bild einer Erzählung, die nicht bloß gut geschriebene Unterhaltungsware ist, sondern die mit spezifisch literarischen Mitteln zu einer zeitgemäßen und historisch adäquaten Neuaneignung der Grande Guerre beiträgt.

58 Vgl. dazu prägnant Starobinski 1976. 


\section{Literatur}

\section{Textausgaben}

Audoin-Rouzeau, Stéphane (2013): Quelle historie. Un récit de filiation (1914-2014), Paris, Seuil/Gallimard.

Barbusse, Henri (2011): Le feu. Journal d'une escouade, dossier et glossaire réalisés par Pascale Salinier, Paris, Gallimard.

Echenoz, Jean (2010): „Entretien“, in: Siècle 21. Littératures et société 17, 118-121.

Echenoz, Jean (2012): 14, Paris, Minuit.

Hugo, Victor (1976): L'CFuvre complète, t. 14: Quatre-vingt-treize, Paris, Jean Bonnot.

Perec, Georges (1989): L'infra-ordinaire, Paris, Seuil.

\section{Forschung}

Adinolfi, Pierangela (2014): „La première guerre mondiale dans le roman français de l'entredeux-guerres. Montherlant, Cocteau, Radiguet“, in: RiCognizini. Rivista di lingue, letterature e culture moderne dell'Università degli Studi di Torino 1, 29-39.

Assmann, Aleida (2016): „Erlebte, erinnerte und erzählte Geschichte“, in: Axel Rüth/Michael Schwarze (Hg.), Erfahrung und Referenz: Erzählte Geschichte im 20. Jahrhundert, Paderborn, Fink, 43-58.

Audoin-Rouzeau, Stéphane (2008): Combattre. Une anthropologie de la guerre moderne (XIXe-XXIe siècle), Paris, Seuil.

Audoin-Rouzeau, Stéphane/Jean-Jacques Becker (éd.) (2012): Encyclopédie de la Grande Guerre, Paris, Perrin.

Bouchy, Florence (2012): „Objets quotidiens et discours ordinaire. Sur les romans de Jean Echenoz et de Jean-Philippe Toussaint“, in: Michel Braud (éd.), L'art de l'ordinaire, Bandol, Vallongues, 129-134.

Brecht, Christoph (2002): ,,Jamais l'histoire ne sera fixée.' Zur Topik historischen Erzählens im Historismus (Flaubert)“, in: Daniel Fulda/Silvia S. Tschopp (Hg.), Literatur und Geschichte. Ein Kompendium zu ihrem Verhältnis von der Aufklärung bis zur Gegenwart, Berlin/New York, De Gruyter, 411-438.

Chaudier, Stéphane (2014): „'écrivain, son style et son double dans 14 de Jean Echenoz“, in: Luc Fraisse/Éric Wessler (éd.), L'Écrivain et ses doubles. Le personnage autoréflexif dans la littérature européenne, Paris, Classiques Garnier, 173-195.

Dugast, Francine (2009): „Le nouveau roman et l'histoire“, in: Dominique Viart (éd.), Nouvelles écritures littéraires de l'Histoire, Paris, Lettres Modernes Minard, 41-72.

Englund, Peter (2011): The Beauty and the Sorrow: An Intimate History of the First World War, New York, Knopf.

Faria, Dominique (2013): „La réception des ,nouveaux auteurs de Minuit‘ (1980-2012)“, in: Intercâmbio 6, $2^{\mathrm{e}}$ série, 65-75. http://ler.letras.up.pt/uploads/ficheiros/11350.pdf [31. 10. 2016]. 
Föcking, Marc (2002): „L'histoire est un roman dont le peuple est l'auteur. Organische Geschichtsschreibung und Faktizität des Romans in Alfred de Vignys Réflexions sur la vérité dans l'art (1829)“, in: Zeitschrift für französische Sprache und Literatur 112, 139-154.

Fulda, Daniel (2002): „Strukturanalytische Hermeneutik. Eine Methode zur Korrelation von Geschichte und Textverfahren“, in: ders./Silvia S. Tschopp (Hg.), Literatur und Geschichte. Ein Kompendium zu ihrem Verhältnis von der Aufklärung bis zur Gegenwart, Berlin/New York, De Gruyter, 39-59.

Fulda, Daniel (22014): „Historiographic Narration“, in: Peter Hühn/Jan Christoph Meister/ John Pier/Wolf Schmid (eds.), Handbook of Narratology, Bd. 1, Berlin/Boston, De Gruyter, 227-240.

Gauyat, Pierre (2009): „La Grande Guerre vue par les auteurs de Romans policiers“, in: Dominique Viart (éd.), Nouvelles écritures littéraires de l'Histoire, Paris, Lettres Modernes Minard, 107-128.

Genette, Gérard (1972): Figures III, Paris, Seuil.

Hartog, François (2003): Régimes d'historicité. Présentisme et expérience du temps, Paris, Seuil.

Isenschmid, Andreas (2014): „Heilige Einfalt! Jean Echenoz, Meister der Raffinesse, scheitert am Ersten Weltkrieg“, in: Die Zeit vom 27. 3. 2014.

Jäger, Ludwig (2008): „Indexikalität und Evidenz. Skizze zum Verhältnis von referentieller und inferentieller Bezugnahme“, in: Horst Wenzel/Ludwig Jäger (Hg.), Deixis und Evidenz, Freiburg, Rombach, 289-315.

Kablitz, Andreas (2006): „Geschichte - Tradition - Erinnerung? Wider die Subjektivierung der Geschichte“, in: Geschichte und Gesellschaft 32, 220-237.

Kaempfer, Jean (1998): Poétique du récit de guerre, Paris, Corti.

Kretschmann, Carsten (2014): „Die politische Funktion der Weltkriegsliteratur in Deutschland und Frankreich 1918 bis 1933/40“, in: Wolfram Pyta/Jörg Lehmann (Hg.), Krieg erzählen raconter la guerre. Darstellungsverfahren in Literatur und Historiographie nach den Kriegen von 1870/71 und 1914/18, Berlin, LIT Verlag, 143-151.

Lindner-Wirsching, Almut (2004): Französische Schriftsteller und ihre Nation im Ersten Weltkrieg, Tübingen, Niemeyer.

Lindner-Wirsching, Almut (2011): „Krieg als ,nationale Erfahrung“ in der deutschen und französischen Erzählliteratur des Ersten Weltkriegs“, in: Wolfram Pyta/Carsten Kretschmann (Hg.), Burgfrieden und Union sacrée. Literarische Deutungen und politische Ordnungsvorstellungen in Deutschland und Frankreich 1914-1933, München, Oldenbourg, 159-177.

Mikowski, Sylvie (2015): „,A Long Long Way“ de Sebastian Barry et ,14` de Jean Echenoz. La mise en crise du roman de la Première Guerre en France et en Irlande“, in: Transversalités 132, 27-42.

Nünning, Ansgar (1995): Von historischer Fiktion zu historiographischer Metafiktion, Bd. 1: Theorie, Typologie und Poetik des historischen Romans, Trier, Wissenschaftlicher Verlag Trier.

Nünning, Ansgar (1999): „,Verbal Fictions‘. Kritische Überlegungen und narratologische Alternativen zu Hayden Whites Einebnung des Gegensatzes zwischen Historiographie und Literatur“, in: Literaturwissenschaftliches Jahrbuch 40, 351-380.

Oster-Stierle, Patricia (2016): „La Grande Guerre et après? Jean Echenoz et Michèle Audin face au savoir historique“, in: Wolfgang Asholt/Ursula Bähler (Hg.), Le savoir historique du roman contemporain, 133-146.

Pavel, Thomas (2003): La Pensée du Roman, Paris, Gallimard. 
Quinn, Tom (2003): „Rewriting Memory: The Great War in Céline’s Voyage au bout de la nuit“, in: Edric Caldicott/Anne Fuchs (eds.), Cultural Memory. Essays on European Literature and History, Oxford, Peter Lang, 343-359.

Rehage, Philipp (2001): „,Badinage belliciste‘? Le rôle de la guerre dans Thomas l'imposteur“, in: David Gullentops (éd.), Jean Cocteau 3: écriture et création, Paris, Lettres Modernes Minard, 141-151.

Ricœur, Paul (2000): La mémoire, l'histoire, l'oubli, Paris, Seuil.

Rubino, Gianfranco (2009): „La Grande Guerre en perspective 2000“, in: Dominique Viart (éd.), Nouvelles écritures littéraires de l'Histoire, Paris, Lettres Modernes Minard, 129-146.

Rubino, Gianfranco/Dominique Viart (éd.) (2014): Le roman français contemporain face à l'histoire. Thèmes et formes, Macerata, Quodlibet.

Rüth, Axel/Michael Schwarze (Hg.) (2016): Erfahrung und Referenz. Erzählte Geschichte im 20. Jahrhundert, Paderborn, Fink.

Schaeffer, Jean-Marie (22014): „Fictional vs. Factual Narration“, in: Peter Hühn/Jan Christoph Meister/John Pier/Wolf Schmid (eds.), Handbook of Narratology, Bd. 1, Berlin/Boston, De Gruyter, 179-196.

Schoentjes, Pierre (2009): Fictions de la Grande Guerre. Variations littéraires sur 14 - 18, Paris, Classiques Garnier.

Schoentjes, Pierre (2012): „14 de Jean Echenoz. Un dernier compte à régler avec la Grande Guerre“, in: Critique 786, 964-981.

Schoentjes, Pierre (2014): „Regards romanesques sur la Grande Guerre. Echenoz, Vuillard et les arts visuels“, in: Gianfranco Rubino/Dominique Viart (éd.), Le roman français contemporain face à l'histoire. Thèmes et formes, Macerata, Quodlibet, 259-274.

Schwarze, Michael (2016): „Latenzerfahrung. Der Algerienkrieg in Laurent Mauvigniers ,Des hommes“ (2009)“, in: Axel Rüth/Michael Schwarze (Hg.), Erfahrung und Referenz. Erzählte Geschichte im 20. Jahrhundert, Paderborn, Fink, 249-271.

Schwarze, Michael: „Psicologia narrativa nei diari di guerra di Jünger e Gadda“, in: Gino Tellini (ed.), In trincea. Gli scrittori alla Grande Guerra, Firenze, Società Editrice Fiorentina [im Druck].

Sermier, Emilien (2013): Variations sur un standard. Jeux et métamorphoses dans les trois romans biographiques de Jean Echenoz, Lausanne, Archipel.

Starobinski, Jean (1976): „Le discours maniaque“, in: Wolfgang Preisendanz/Rainer Warning (Hg.), Das Komische, München, Fink, 383-384.

Tengelyi, László (2010): „In Verteidigung der Geschichtserfahrung. Zur Auseinandersetzung von Paul Ricœur und Hayden White“, in: Thiemo Breyer/Daniel Creutz (Hg.), Erfahrung und Geschichte. Historische Sinnbildung im Pränarrativen, Berlin/New York, De Gruyter, 185-199.

Theeten, Griet (2009): La Grande Guerre en fiction. La représentations de la Première guerre mondiale dans la littérature française de l'extrême contemporain, Gent, Gent University.

Viart, Dominique (2000): „Écrire au présent: l'esthétique contemporaine“, in: Michèle Touret/Francine Dugast-Porte (éd.), Le temps des lettres. Quelles périodisations pour l'historie de la littérature française du XXe siècle?, Rennes, Presses Universitaires de Rennes, 317-336.

Viart, Dominique (2009): „Nouveaux modèles de représentation de l'histoire en littérature contemporaine“, in: id. (éd.), Nouvelles écritures littéraires de l'Histoire, Paris, Lettres Modernes Minard, 11-39. 
Warning, Rainer (2001): „Erzählen im Paradigma. Kontingenzbewältigung und Kontingenzexposition“, in: Romanistisches Jahrbuch 52, 176-209.

White, Hayden (1973): Metahistory. The Historical Imagination in 19th Century Europe, Baltimore/London, Johns Hopkins Univ. Press.

White, Hayden (1978): Tropics of discourse: Essays in Cultural Criticism, Baltimore/London, Johns Hopkins Univ. Press.

Wischermann, Clemens (2015): „Mensch-Tier-Beziehungen in Kriegen des 20. Jahrhunderts“, in: Michael Jonas/Ulrich Lappenküper/Oliver von Wrochem (Hg.), Dynamiken der Gewalt. Krieg im Spannungsgeld von Politik, Ideologie und Gesellschaft, Paderborn, Schöningh, 359-376. 
